\title{
Pre-fertilization Barrier, Crossability and Meiotic Behavior of Interspecific Hybrids Among Brassica Species
}

\author{
Usha Pant ( $\square$ ushapantgpb@gmail.com ) \\ G B Pant University of Agriculture and Technology: Govind Ballabh Pant University of Agriculture \& Technology \\ Shikha Joshi \\ G B Pant University of Agriculture and Technology: Govind Ballabh Pant University of Agriculture \& Technology \\ Preeti Lohani \\ G B Pant University of Agriculture and Technology: Govind Ballabh Pant University of Agriculture \& Technology \\ Neha Dahiya \\ G B Pant University of Agriculture and Technology: Govind Ballabh Pant University of Agriculture \& Technology
}

\section{Research Article}

Keywords: Interspecific hybridization, B. carinata, Pre-fertilization barrier, meiotic abnormalities

Posted Date: August 23rd, 2021

DOI: https://doi.org/10.21203/rs.3.rs-791559/v1

License: (c) This work is licensed under a Creative Commons Attribution 4.0 International License. Read Full License 


\section{Abstract}

Inter-specific hybridization is an important driving force in plant evolution, speciation and creation of novel genetic variations those are not available with the naturally occurring species. Present investigation includes study of pre-fertilization barriers, phenotypic characterization and the meiotic abnormalities during microsporogenesis in the interspecific hybrids. Mean pollen tube germination percent in interspecific hybrids was recorded to be highest in case of $B$. carinata $x$ B. nigra (33.67 \pm 11.35$)$ and lowest between $B$. carinata $X$ B. napus $(18.68 \pm 6.62)$ whereas in the reciprocal crosses it was highest for $B$. napus $x$ B. carinata (33.06 \pm 13.41 ). Siliqua set ranged from $33 \%$ in $B$. carinata $x B$. napus to $72 \%$ in selfed $B$. carinata. Seed set $\%$ ranged from $0 \%$ in $B$. rapa $\times$ B. carinata and B.nigra $\times$ B.carinatato $25.64 \%$ for B. carinata $\times$ B.nigra. Mean pollen tube growth was recorded highest in $B$. carinata $x$ B. nigra (21.23 \pm 5.33$)$ while was lowest in $B$. carinata $x B$. napus $(10.18 \pm 3.17)$ and among reciprocals it was highest for $B$. nigra $x B$. carinata (21.39 \pm 7.53). Pollen tube abnormalities viz., coiling and bending of pollen tube, hairpin shaped pollen tube, swelling of tube tip, tube bifurcation, more than one tube emerging from pollen, tubes growing in wrong direction were observed. $B$. carinata as pollen parent showed highest mean pollen tube abnormality percent with $B$. napus (36.32 \pm 8.62 ) whereas lowest with $B$. nigra (18.09 \pm 6.73$)$. It was observed highest with $B$. rapa (49.170 \pm 13.55$)$ when $B$. carinata was used as pollen parent. Some of the pre fertilization barriers related to stylar and ovule region can also be observed through fluroscent microscopy. As per the observations $B$. carinata performed better as a seed parent with $B$. nigra, $B$. juncea and $B$. rapa whereas, with $B$. napus it performed good as a pollen parent. Correlation study gave the association the above traits with each other. The meiotic abnormalities showed improper alignment at M-I, M-II, A-I, A-II and Telophase.

\section{Introduction}

Brassica is one of the genuses belonging to the family Brassicaceae and includes crops of agricultural and horticultural importance. Out the species under genus Brassica, six are of economic importance viz., B.rapa (AA), B.nigra (BB), B.oleraceae (CC), B.juncea (AABB), B.napus (AACC), and B.carinata (BBCC). Out of these, the diploid species of Brassica are considered to be the progenitor species or primary species whose natural cross combinations followed by chromosome doubling resulted in evolution of three amphidiploids secondary species. The genetic relationship between these primary and secondary species was depicted through U's Triangle Model (Nagaharu U., 1935). Rapeseed mustard is one of the most important oilseed crop grown worldwide. Oilseed Brassica is the second most important edible oilseed crop in India after soybean and accounts for nearly one third of the oil produced in India, making it the country's key edible oilseed crop. Wide or distant hybridization is a mating between individuals of different species or genera. It combines different genomes into one nucleus and thus results in changes in genotypes and phenotypes of the progenies. Since, the first ever interspecific hybrid reported by Thomas Fairchild in 1717, thousands of interspecific crosses have been attempted to improve the quality of cultivated species. Interspecific hybridization is a very important tool in creation of novel genetic variability and in transmission of agronomically important characters from progenitor to the cultivated species. Resynthesis of Brassica is usually undertaken for exploiting the wider gene pool of the diploids.Furthermore, the cytogenetical studies of chromosome pairing in the meiotic divisions of the wide hybrids helps in identifying the genomic affinities and the phylogenetic relationships of the diverse genome. In Brassica genus interspecific and intergeneric hybridizations have been extensively performed all over the world between the cultivated species and wild relatives to develop more potentially useful cultivars with improved biotic and abiotic stress tolerance.

But not all of the crosses are successful, the cross incompatibility in the interspecific hybridizations has impeded the development of hybrids. Many efforts have been made to improve the Brassica crops by sexual crosses intended to enrich the germplasm through artificial resynthesis and wide hybridization of naturally existing amphidiploids. With due course of time, the cultivated primary species have been improved to a great extent for their agronomic performance and their genome have also been modified considerably. Similarly the primary genome residing in the amphidilpoids have also been undergone cytological changes and the modified. Therefore interspecific hybridization among the existing primary and secondary species might bring some novel variant that enrich the available germplasm. However, even though there is large tolerance for interspecific hybridization between the Brassica crop species and several of the wild species related to the Brassica species, successful use of sexual hybridization is limited. A number of preand post-fertilization barriers limit the exploration of diversity of different genomic combination. Depending upon the types of barriers several methods have been used to overcome these barriers.

\section{Material And Methods}

Five species of Brassica were used for interspecific hybridization i.e., B. rapa, B. nigra, B. juncea, B. napus, $B$. carinata. Eight buds per raceme were emasculated in the evening hours and were then pollinated the next day in the morning hours. All the possible crosses among the 5 species were made taking the reciprocals also into consideration. Selfing of all the 5 species was also carried out. For understanding pollen pistil interaction pre-fertilization studies were made for which bright field microscopy and fluorescent microscopy at different intervals after pollination were carried out. For bright field microscopy the pistil were removed from the raceme carefully and were kept in $1 \mathrm{~N} \mathrm{HCL}$ for $10 \mathrm{~min}$. After that the buds were rinsed with distilled water and stained with $1 \%$ aniline blue for $5-15 \mathrm{sec}$. After staining the samples were put in destaining solution for 30 min. After destaining the samples were rinsed with distilled water and mounted on pure lactic acid. For fluroscent microscopy Aniline Blue was used as a callose staining flurochrome (Dumas and Knox, 1983). The fixed samples were rinsed with distilled water and after careful maceration squash preparation was done. Prior to squash preparation the samples were kept in $8 \mathrm{~N} \mathrm{NaOH}$ solution for about 2 to 6 hours for softening. The samples were then stained in Decolourised Aniline Blue (DAB) solution for $30 \mathrm{~min}$ to 1 hour. Parameters viz., Pollen germination percent, mean pollen tube growth and pollen tube abnormality percent were assessed. Siliqua set percent and seed set percent were also recorded for assessing cross compatibility relationship. Morphological studies of the hybrids were also done in the field along with the parents and observation on different morphological characters was taken. Meiosis study of the F1s 
was also carried out and for this the buds were collected in the morning and were fixed in Carnoy's solution for 24 hours. After that the samples were transferred to $70 \%$ ethanol and refrigerated till further use. For slide preparation the anther was crushed and one percent acetocarmine was used to stain it. Correlation between the crossability parameters was also studied.

\section{Results}

\section{(a) Cross compatibility relationship:}

Crossability relationship among brassica species was determined on the basis of successful siliqua and seed set percent. The direct inter-specific cross involving diploid and amphidiploids and their reciprocals were included for the study. Siliqua set percent in inter-specific hybrids ranged from $21-61 \%$. It was recorded highest in the interspecific cross of $B$. napus $\times$ B. juncea $(61 \%)$ whereas lowest in the interspecific cross of $B$. nigra $\times B$. napus ( $21 \%)$. In inter-specific crosses seed set percent ranged from $0.00 \%$ to $28.89 \%$. Highest seed set percent was recorded in the cross $B$. juncea $\times B$. napus (28.89\%). However, no seed set was observed in the crosses of $B$. rapa $\times$ B. napus, B. rapa $\times$ B. carinata, B. nigra $\times$ B. carinata, B. nigra $\times$ B. napus (Table 1$)$. The crossability relationship based on seed set percentage is depicted in Fig. 1. It is reflected from the results that in case diploid and amphidiploids interspecific crosses mostly the higher siliqua and seed set were observed where the amphidiploids were taken as female parent. In most of the interspecific crosses involving amphidiploids higher seed and siliqua set was observed when a parent with higher chromosome number is taken as female. In the crosses where only diploids were used i.e. B. rapa and B. nigra similar effect of female parent was found. Higher siliqua and seed set has been observed in cross having B. nigra as female parent. Significant role of female parent in controlling the seed set has also been reported by saba (2012). The genome affinity during interspecific hybridization is key factor for the success of crosses. The genomes that are close and common gave better result as compare to the distant genome (Katche et al., 2019). The genome affinity has extensively studied earlier and later on validated through molecular cytogenetic studies in Brassicas. Among Brassica crosses mostly the amphidiploids with higher chromosome number taken as female gave better result while in case of crosses involving only diploid species with high chromosome number serve better as female. 
Table 1

Seed Set $\%$ and Siliqua Set $\%$ of the crosses

\begin{tabular}{|lll|}
\hline Cross & Siliqua set \% & Seed set \% \\
\hline B. rapaselfed & 71.67 & 82.00 \\
\hline B. rapa $\times$ B. nigra & 31.67 & 2.00 \\
\hline B. rapa $\times$ B. juncea & 41.67 & 2.50 \\
\hline B. rapa $\times$ B. napus & 35.00 & 0.00 \\
\hline B. rapa $\times$ B. carinata & 41.00 & 0.00 \\
\hline B. nigra selfed & 74.00 & 79.00 \\
\hline B. nigra $\times$ B. rapa & 36.00 & 15.30 \\
\hline B. nigra $\times$ B. juncea & 48.33 & 18.70 \\
\hline B. nigra $\times$ B. napus & 21.00 & 0.00 \\
\hline B. nigra $\times$ B. carinata & 36.67 & 0.00 \\
\hline B. junceaselfed & 82.00 & 81.00 \\
\hline B. juncea $\times$ B. rapa & 55.10 & 13.33 \\
\hline B. juncea $\times$ B. nigra & 49.36 & 8.87 \\
\hline B. juncea $\times$ B. napus & 40.00 & 28.89 \\
\hline B. juncea $\times$ B. carinata & 36.67 & 6.67 \\
\hline B. napus selfed & 71.00 & 75.00 \\
\hline B. napus $\times$ B. rapa & 53.00 & 27.77 \\
\hline B. napus $\times$ B. nigra & 31.00 & 13.89 \\
\hline B. napus $\times$ B. juncea & 61.00 & 7.40 \\
\hline B. napus $\times$ B. carinata & 54.39 & 16.50 \\
\hline B. carinataselfed & 72.00 & 79.00 \\
\hline B. carinata $\times$ B. rapa & 33.00 & 10.65 \\
\hline B. carinata $\times$ B. nigra & 74.00 & 25.64 \\
\hline B. carinata $\times$ B. juncea & 58.33 & 2.17 \\
\hline B. carinata $\times$ B. napus & 27.00 & 4.49 \\
\hline
\end{tabular}


Table 2

Pollen germination\%, Pollen tube growth $(\mu)$ and Pollen abnormality\% at different time intervals after pollination.

\begin{tabular}{|c|c|c|c|c|c|c|c|c|}
\hline Cross & Parameters & 30min & $2 \mathrm{hr}$ & $8 \mathrm{hr}$ & $18 \mathrm{hr}$ & $24 \mathrm{hr}$ & $48 \mathrm{hr}$ & Mean \\
\hline \multirow[t]{3}{*}{ B. rapa (selfed) } & Pollen germination percent (\%) & 5.34 & 43.00 & 55.78 & 77.07 & 78.60 & 80.45 & $56.70 \pm 11.95$ \\
\hline & Pollen tube growth $(\mu)$ & 8.23 & 14.20 & 20.23 & 35.67 & 56.78 & 79.49 & $35.77 \pm 11.28$ \\
\hline & Pollen abnormality percent (\%) & 00.00 & 00.00 & 00.00 & 00.00 & 00.00 & 00.00 & 00.00 \\
\hline \multirow[t]{3}{*}{ B. rapa $\times$ B. nigra } & Pollen germination percent (\%) & 0.00 & 0.00 & 40.00 & 41.67 & 42.10 & 45.45 & $28.20 \pm 8.95$ \\
\hline & Pollen tube growth $(\mu)$ & 0.00 & 5.71 & 8.01 & 12.45 & 13.34 & 21.66 & $10.20 \pm 3.03$ \\
\hline & Pollen abnormality percent (\%) & 0.00 & 38.00 & 47.00 & 50.00 & 54.00 & 88.00 & $46.17 \pm 11.58$ \\
\hline \multirow[t]{3}{*}{ B. rapa $\times$ B. juncea } & Pollen germination percent (\%) & 0.00 & 23.00 & 33.56 & 48.00 & 51.25 & 60.60 & $36.07 \pm 9.04$ \\
\hline & Pollen tube growth $(\mu)$ & 0.00 & 5.71 & 17.56 & 28.56 & 38.00 & 53.80 & $23.94 \pm 8.28$ \\
\hline & Pollen abnormality percent (\%) & 0.00 & 33.00 & 44.00 & 48.00 & 52.00 & 57.00 & $39.00 \pm 8.48$ \\
\hline \multirow[t]{3}{*}{ B. rapa $\times$ B. napus } & Pollen germination percent (\%) & 0.00 & 32.00 & 33.33 & 43.00 & 50.00 & 50.00 & $34.72 \pm 7.64$ \\
\hline & Pollen tube growth $(\mu)$ & 0.00 & 8.93 & 18.30 & 26.90 & 26.89 & 48.35 & $21.56 \pm 6.85$ \\
\hline & Pollen abnormality percent (\%) & 0.00 & 31.00 & 43.10 & 65.78 & 33.33 & 50.00 & $37.20 \pm 9.04$ \\
\hline \multirow[t]{3}{*}{ B.rapa x B.carinata } & Pollen germination percent (\%) & 0.00 & 0.00 & 23.34 & 25.00 & 39.19 & 64.70 & $25.37 \pm 10.05$ \\
\hline & Pollen tube growth $(\mu)$ & 0.00 & 6.67 & 6.70 & 10.92 & 10.92 & 11.01 & $7.7 \pm 1.76$ \\
\hline & Pollen abnormality percent (\%) & 0.00 & 6.67 & 83.33 & 25.00 & 80.00 & 40.00 & $49.17 \pm 13.55$ \\
\hline \multirow[t]{3}{*}{ B. nigra (selfed) } & Pollen germination percent (\%) & 8.00 & 23.00 & 45.00 & 56.63 & 74.59 & $98 . .36$ & $51.03 \pm 13.61$ \\
\hline & Pollen tube growth $(\mu)$ & 1.78 & 11.47 & 29.67 & 31.56 & 49.70 & 76.89 & $33.51 \pm 11.04$ \\
\hline & Pollen abnormality percent (\%) & 00.00 & 00.00 & 00.00 & 00.00 & 00.00 & 00.00 & 00.00 \\
\hline \multirow[t]{3}{*}{ B. nigra $\times$ B. rapa } & Pollen germination percent (\%) & 0.00 & 0.00 & 23.00 & 33.57 & 41.55 & 42.56 & $23.45 \pm 7.69$ \\
\hline & Pollen tube growth $(\mu)$ & 0.00 & 6.34 & 10.00 & 16.00 & 20.15 & 21.78 & $12.38 \pm 2.45$ \\
\hline & Pollen abnormality percent (\%) & 0.00 & 0.00 & 57.00 & 66.90 & 78.00 & 84.00 & $47.65 \pm 15.54$ \\
\hline \multirow[t]{3}{*}{ B. nigra $\times$ B. juncea } & Pollen germination percent (\%) & 0.00 & 15.98 & 43.56 & 48.86 & 58.79 & 66.90 & $39.02 \pm 10.55$ \\
\hline & Pollen tube growth $(\mu)$ & 0.00 & 8.90 & 26.56 & 32.56 & 42.34 & 59.50 & $28.31 \pm 8.89$ \\
\hline & Pollen abnormality percent (\%) & 0.00 & 0.00 & 50.00 & 50.00 & 54.00 & 56.20 & $35.03 \pm 11.12$ \\
\hline \multirow[t]{3}{*}{ B. nigra $\times$ B. napus } & Pollen germination percent (\%) & 0.00 & 0.00 & 0.00 & 18.34 & 21.50 & 26.40 & $11.04 \pm 5.05$ \\
\hline & Pollen tube growth $(\mu)$ & 0.00 & 9.00 & 9.06 & 11.78 & 13.67 & 15.70 & $9.87 \pm 2.24$ \\
\hline & Pollen abnormality percent (\%) & 0.00 & 0.00 & 68.00 & 73.00 & 78.60 & 78.66 & $49.71 \pm 15.80$ \\
\hline \multirow[t]{3}{*}{ B.nigra $\times$ B.carinata } & Pollen germination percent (\%) & 0.00 & 21.67 & 33.00 & 33.00 & 50.00 & 50.00 & $31.28 \pm 7.10$ \\
\hline & Pollen tube growth $(\mu)$ & 0.00 & 2.90 & 17.56 & 24.56 & 36.32 & 47.00 & $21.39 \pm 7.53$ \\
\hline & Pollen abnormality percent (\%) & 0.00 & 0.00 & 22.22 & 32.78 & 42.16 & 62.87 & $26.67 \pm 10.05$ \\
\hline \multirow[t]{3}{*}{ B.carinata (selfed) } & Pollen germination percent (\%) & 1.23 & 2.46 & 27.11 & 51.44 & 87.00 & 94.60 & $43.97 \pm 16.64$ \\
\hline & Pollen tube growth $(\mu)$ & 1.23 & 8.50 & 16.09 & 27.67 & 40.90 & 42.00 & $22.73 \pm 6.92$ \\
\hline & Pollen abnormality percent (\%) & 00.00 & 00.00 & 00.00 & 00.00 & 00.00 & 00.00 & 00.00 \\
\hline \multirow[t]{3}{*}{ B.carinata $\times$ B. rapa } & Pollen germination percent (\%) & 0.00 & 8.23 & 13.56 & 23.67 & 45.12 & 54.67 & $24.21 \pm 8.79$ \\
\hline & Pollen tube growth $(\mu)$ & 0.00 & 21.00 & 21.54 & 23.56 & 21.78 & 26.45 & $19.06 \pm 3.9$ \\
\hline & Pollen abnormality percent (\%) & 0.00 & 0.00 & 0.00 & 85.71 & 50.00 & 60.00 & $32.62 \pm 15.34$ \\
\hline \multirow[t]{3}{*}{ B.carinata $\times$ B.nigra } & Pollen germination percent (\%) & 0.00 & 11.18 & 23.76 & 44.40 & 45.78 & 76.89 & $33.67 \pm 11.35$ \\
\hline & Pollen tube growth $(\mu)$ & 0.00 & 14.87 & 18.40 & 26.40 & 31.70 & 36.00 & $21.23 \pm 5.33$ \\
\hline & Pollen abnormality percent (\%) & 0.00 & 8.70 & 11.90 & 12.70 & 30.00 & 45.21 & $18.09 \pm 6.73$ \\
\hline B.carinata $\times$ B.juncea & Pollen germination percent (\%) & 0.00 & 10.00 & 22.67 & 34.80 & 56.70 & 67.01 & $31.86 \pm 10.71$ \\
\hline
\end{tabular}




\begin{tabular}{|c|c|c|c|c|c|c|c|c|}
\hline Cross & Parameters & $30 \mathrm{~min}$ & $2 \mathrm{hr}$ & $8 \mathrm{hr}$ & $18 \mathrm{hr}$ & $24 \mathrm{hr}$ & $48 \mathrm{hr}$ & Mean \\
\hline & Pollen tube growth $(\mu)$ & 0.00 & 10.90 & 13.90 & 23.80 & 34.10 & 36.00 & $19.78 \pm 5.74$ \\
\hline & Pollen abnormality percent (\%) & 0.00 & 15.00 & 21.87 & 22.60 & 37.80 & 44.60 & $23.65 \pm 6.53$ \\
\hline \multirow[t]{3}{*}{ B.carinata $\times$ B.napus } & Pollen germination percent (\%) & 0.00 & 0.00 & 18.65 & 21.34 & 34.00 & 38.09 & $18.68 \pm 6.62$ \\
\hline & Pollen tube growth $(\mu)$ & 0.00 & 5.65 & 5.74 & 12.56 & 16.14 & 21.00 & $10.18 \pm 3.17$ \\
\hline & Pollen abnormality percent (\%) & 0.00 & 35.00 & 64.98 & 33.50 & 40.21 & 44.21 & $36.32 \pm 8.62$ \\
\hline \multirow[t]{3}{*}{ B. juncea (sef) } & Pollen germination percent (\%) & 3.97 & 22.23 & 34.67 & 46.89 & 78.00 & 95.66 & $46.90 \pm 14.08$ \\
\hline & Pollen tube growth $(\mu)$ & 2.32 & 10.65 & 25.00 & 38.56 & 55.00 & 56.97 & $31.42 \pm 9.27$ \\
\hline & Pollen abnormality percent (\%) & 00.00 & 00.00 & 00.00 & 00.00 & 00.00 & 00.00 & 00.00 \\
\hline \multirow[t]{3}{*}{ B. juncea $\times$ B. rapa } & Pollen germination percent (\%) & 0.00 & 22.78 & 28.56 & 53.00 & 55.64 & 62.00 & $37.00 \pm 9.78$ \\
\hline & Pollen tube growth $(\mu)$ & 0.00 & 7.79 & 16.78 & 36.67 & 50.00 & 53.47 & $27.45 \pm 9.17$ \\
\hline & Pollen abnormality percent (\%) & 0.00 & 0.00 & 18.00 & 23.00 & 55.00 & 55.00 & $21.23 \pm 8.48$ \\
\hline \multirow[t]{3}{*}{ B. juncea $\times$ B. nigra } & Pollen germination percent (\%) & 0.00 & 12.80 & 27.61 & 47.45 & 57.32 & 68.34 & $35.59 \pm 10.86$ \\
\hline & Pollen tube growth $(\mu)$ & 0.00 & 9.38 & 12.39 & 29.67 & 32.56 & 44.04 & $21.34 \pm 6.80$ \\
\hline & Pollen abnormality percent (\%) & 0.00 & 0.00 & 33.33 & 55.56 & 36.36 & 36.36 & $22.72 \pm 9.21$ \\
\hline \multirow[t]{3}{*}{ B. juncea $\times$ B. napus } & Pollen germination percent (\%) & 0.00 & 0.00 & 24.00 & 42.00 & 54.85 & 68.00 & $31.48 \pm 11.59$ \\
\hline & Pollen tube growth $(\mu)$ & 0.00 & 20.00 & 22.35 & 24.34 & 30.28 & 42.00 & $23.165 \pm 5.64$ \\
\hline & Pollen abnormality percent (\%) & 0.00 & 0.00 & 29.49 & 56.50 & 74.00 & 74.00 & $37.54 \pm 13.34$ \\
\hline \multirow[t]{3}{*}{ B.juncea x B.carinata } & Pollen germination percent (\%) & 0.00 & 0.00 & 19.80 & 19.80 & 51.00 & 65.00 & $29.80 \pm 11.16$ \\
\hline & Pollen tube growth $(\mu)$ & 0.00 & 8.34 & 10.32 & 24.67 & 32.00 & 46.62 & $20.33 \pm 7.08$ \\
\hline & Pollen abnormality percent (\%) & 0.00 & 0.00 & 16.67 & 80.00 & 76.04 & 67.12 & $39.97 \pm 15.68$ \\
\hline \multirow[t]{3}{*}{ B. napus (selfed) } & Pollen germination percent (\%) & 1.23 & 41.00 & 56.00 & 68.91 & 75.00 & 90.56 & $55.45 \pm 12.84$ \\
\hline & Pollen tube growth $(\mu)$ & 1.67 & 18.60 & 32.56 & 45.70 & 48.60 & 52.90 & $33.34 \pm 8.14$ \\
\hline & Pollen abnormality percent (\%) & 00.00 & 00.00 & 00.00 & 00.00 & 00.00 & 00.00 & 00.00 \\
\hline \multirow[t]{3}{*}{ B.napus x B.carinata } & Pollen germination percent (\%) & 0.00 & 0.00 & 16.67 & 40.00 & 66.67 & 75.00 & $33.06 \pm 13.44$ \\
\hline & Pollen tube growth $(\mu)$ & 0.00 & 10.83 & 11.55 & 16.30 & 16.00 & 21.65 & $12.72 \pm 3.00$ \\
\hline & Pollen abnormality percent (\%) & 0.00 & 0.00 & 47.21 & 40.30 & 20.96 & 32.79 & $23.54 \pm 8.25$ \\
\hline \multirow[t]{3}{*}{ B. napus $\times$ B. rapa } & Pollen germination percent (\%) & 0.00 & 20.00 & 35.29 & 40.00 & 50 & 85.71 & $38.50 \pm 11.83$ \\
\hline & Pollen tube growth $(\mu)$ & 0.00 & 11.66 & 28.67 & 36.78 & 46.91 & 54.40 & $29.74 \pm 8.50$ \\
\hline & Pollen abnormality percent (\%) & 0.00 & 8.09 & 23.00 & 38.00 & 64.00 & 66.67 & $33.29 \pm 11.44$ \\
\hline \multirow[t]{3}{*}{ B. napus $\times$ B. nigra } & Pollen germination percent (\%) & 0.00 & 9.09 & 18.18 & 25.00 & 25.00 & 28.57 & $17.64 \pm 4.52$ \\
\hline & Pollen tube growth $(\mu)$ & 0.00 & 7.25 & 7.34 & 7.46 & 10.76 & 16.09 & $8.15 \pm 2.15$ \\
\hline & Pollen abnormality percent (\%) & 0.00 & 0.00 & 50.00 & 50.00 & 75.00 & 76.89 & $41.98 \pm 14.10$ \\
\hline \multirow[t]{3}{*}{ B. napus $\times$ B. juncea } & Pollen germination percent (\%) & 0.00 & 27.78 & 47.40 & 55.00 & 55.87 & 68.00 & $42.34 \pm 10.05$ \\
\hline & Pollen tube growth $(\mu)$ & 0.00 & 7.50 & 7.89 & 14.65 & 17.89 & 22.50 & $11.74 \pm 3.33$ \\
\hline & Pollen abnormality percent (\%) & 0.00 & 0.00 & 0.00 & 75.00 & 33.33 & 33.33 & $23.61 \pm 12.25$ \\
\hline
\end{tabular}


Table 3

Correlation between parameters of crossability in different sets of crosses

\begin{tabular}{|c|c|c|c|c|c|c|c|c|c|c|}
\hline $\begin{array}{l}\text { Species } \\
\text { used as } \\
\text { seed } \\
\text { parents } \\
\text { in } \\
\text { crosses } \\
\text { with } \\
\text { other } \\
\text { species }\end{array}$ & $\begin{array}{l}\text { Pollen } \\
\text { germination } \\
\text { percent and } \\
\text { mean } \\
\text { pollen tube } \\
\text { growth }\end{array}$ & $\begin{array}{l}\text { Pollen } \\
\text { germination } \\
\text { percent and } \\
\text { pollen tube } \\
\text { abnormality } \\
\text { percent }\end{array}$ & $\begin{array}{l}\text { Pollen } \\
\text { germination } \\
\text { percent and } \\
\text { siliqua set } \\
\text { percent }\end{array}$ & $\begin{array}{l}\text { Pollen } \\
\text { germination } \\
\text { percent and } \\
\text { seed set } \\
\text { percent }\end{array}$ & $\begin{array}{l}\text { Mean } \\
\text { pollen tube } \\
\text { growth and } \\
\text { pollen tube } \\
\text { abnormality } \\
\text { percent }\end{array}$ & $\begin{array}{l}\text { Mean } \\
\text { pollen } \\
\text { tube } \\
\text { growth } \\
\text { and } \\
\text { siliqua } \\
\text { set } \\
\text { percent }\end{array}$ & $\begin{array}{l}\text { Mean } \\
\text { pollen } \\
\text { tube } \\
\text { growth } \\
\text { and } \\
\text { seed } \\
\text { set } \\
\text { percent }\end{array}$ & $\begin{array}{l}\text { Pollen tube } \\
\text { abnormality } \\
\text { and siliqua } \\
\text { set percent }\end{array}$ & $\begin{array}{l}\text { Pollen tube } \\
\text { abnormality } \\
\text { and seed } \\
\text { set percent }\end{array}$ & $\begin{array}{l}\text { Siliqua } \\
\text { set } \\
\text { percent } \\
\text { and } \\
\text { seed } \\
\text { set } \\
\text { percent }\end{array}$ \\
\hline B. rapa & $0.995^{\star \star}$ & $-0.972^{\star}$ & 0.115 & 0.329 & $-0.965^{\star}$ & 0.204 & 0.279 & 0.10 & -0.14 & 0.26 \\
\hline B. nigra & 0.949 & -0.769 & $0.969 *$ & 0.557 & -0.79 & 0.883 & 0.425 & -0.592 & 0.099 & 0.743 \\
\hline $\begin{array}{l}\text { B. } \\
\text { juncea }\end{array}$ & 0.687 & $-0.989 *$ & $0.993 *$ & -0.157 & -0.57 & 0.739 & 0.289 & $-0.974 *$ & 0.279 & -0.169 \\
\hline $\begin{array}{l}\text { B. } \\
\text { napus }\end{array}$ & $0.997 * *$ & -0.783 & $0.969 *$ & 0.62 & -0.738 & $0.950 *$ & 0.136 & -0.912 & 0.284 & -0.5 \\
\hline $\begin{array}{l}\text { B. } \\
\text { carinata }\end{array}$ & 0.886 & $-0.976^{\star}$ & $0.960 *$ & 0.499 & -0.805 & 0.760 & 0.515 & $-0.997 * \star$ & -0.618 & 0.620 \\
\hline
\end{tabular}

Table 4

Correlation between different parameters of crossability

\begin{tabular}{|c|c|c|c|c|c|}
\hline & $\begin{array}{l}\text { Pollen germination } \\
\text { percent }\end{array}$ & $\begin{array}{l}\text { Mean pollen tube } \\
\text { growth }\end{array}$ & $\begin{array}{l}\text { Pollen tube abnormality } \\
\text { percent }\end{array}$ & $\begin{array}{l}\text { Siliqua set } \\
\text { percent }\end{array}$ & $\begin{array}{l}\text { Seed set } \\
\text { percent }\end{array}$ \\
\hline Pollen germination percent & 1 & $0.884 * \star$ & $-0.623^{\star \star}$ & $0.738 * \star$ & 0.294 \\
\hline Mean pollen tube growth & & 1 & $-0.665^{\star \star}$ & $0.650 * \star$ & 0.411 \\
\hline $\begin{array}{l}\text { Pollen tube abnormality } \\
\text { percent }\end{array}$ & & & 1 & $-0.783^{\star \star}$ & -0.282 \\
\hline Siliqua set percent & & & & 1 & $0.460 *$ \\
\hline Seed set percent & & & & & 1 \\
\hline
\end{tabular}

\section{(b) Study of Pre-fertilization barriers:}

\section{Bright field microscopy}

Bright field microscopy and fluorescent microscopy was carried out to study the pre-fertilization in different inter-specific crosses. The buds fixed under different time intervals of pollination were subjected to microscopy. Through Bright field microscopy, three parameters were estimated viz., pollen germination percent, pollen tube growth and pollen tube abnormality percent. In the self-pollinated samples pollen grains started showing germination 30 minutes after pollination and highest pollen germination percentage at 30 minutes was recorded for $B$. nigra self (8.0\%), while in inter-specific hybrids the pollen germination started 2 hrs or 8 hrs after pollination. The rate of pollen tube growth was almost constant when measured at 2 hrs of pollination and $8 \mathrm{hrs}$ of pollination. However an increase in growth of pollen tube was recorded at $18 \mathrm{hrs}$ and $24 \mathrm{hrs}$ of pollination. A slow growth rate of pollen tube was observed from $24 \mathrm{hrs}$ to $48 \mathrm{hrs}$ of pollination.

\section{Pollen germination percent:}

It is the first step of pollen pistil interaction and was found to be variable for interspecific hybrids and selfing. In Selfing, the Pollen germination percent was found to be highest for $B$. rapa i.e., 35.77 and in interspecific cross it was recorded highest for $B$. napus $\times$ B. juncea i.e., $42.34 \%$ whereas, was lowest for B. nigrax B.napus (11.04\%).

\section{Pollen tube growth:}

Mean pollen tube growth was recorded highest in the cross of $B$. napus $\times B$. rapa(29.74 $)$, while was lowest for $B$. napus $\times$ B.nigra(8.15 $\mu)$. In case of selfing it was highest found highest in $B$. rapa i.e., $35.77 \mu$.

\section{Pollen tube abnormality percent:}

Pollen tube abnormalities viz., coiling and bending of pollen tube, hairpin shaped pollen tube, swelling of tube tip, tube bifurcation, more than one tube emerging from pollen, tubes growing in wrong direction were observed. Highest pollen tube abnormality percent $49.71 \%$ was observed in the cross of $B$. nigra $\times$ B. napus which did not share any genome with each other, whereas, it was lowest for B. carinata $\times$ B. nigra (18.09\%). 
Fluorescent Microscopy: For studying the pollen tube growth in stylar region fluorescent microscopy was done at different time intervals for each and every cross. Breaking of pollen tube in upper half of style, twisting of pollen tubes in lower half of style and pollen tubes not entering the ovules but passing through the gaps in between the ovules were some of the pre fertilization barriers recorded (Fig. 3).

\section{(c) Correlation Studies:}

Correlation studies were carried out between different parameters of crossability and prefertilization barriers, so to determine the relationship between them. The pollen germination percent showed highly significant and positive correlation with mean pollen tube growth, and percent siliqua set and highly significant and negative correlation with pollen tube abnormality percent.A highly significant and positive correlation also existed between mean pollen tube growth and siliqua set percent and a highly significant and negative correlation existed between mean pollen tube growth and pollen tube abnormality percent.Pollen tube abnormality percent had a highly significant and negative correlation with all of the crossability parameters with lack of correspondence with seed set percent.Siliqua set percent was found in correspondence with percent seed set. Affinity of $A$ genome with $C$ genome is more pronounced than affinity of $\mathrm{A}$ genome with $\mathrm{B}$ or $\mathrm{B}$ genome with $\mathrm{C}$ genome. It was reported in the literature that during evolution $\mathrm{A}$ and $\mathrm{C}$ genome had evolved from the same lineage while the B genome has taken a different route. That is why the B genome has showed low affinity with both the two genome (Kaminski et al., 2020).

\section{(d) Morphological characterization of interspecific hybrids:}

The hybrids were morphologicaly assessed and compared to the parents. The morphology of interspecific hybrids was intermediate between both of the parents with the predominance of female in some characters while in $B$. napus $\times B$. juncea and $B$. napus $\times$ B. carinata morphology of the hybrids resembled the female parent. In a cross of $B$. carinata $\times$ B. juncea $\mathrm{F}_{1} s$ were taller and exhibited profuse branching and had more number of siliqua than both of the parents. The siliqua size of hybrids was smaller than that of parents, with poor seed set. The hybrids were partially sterile and so no seed set upon selfing. Seeds were recovered only from open pollination (Fig. 5a). B.carinata $x$ B. rapa $\mathrm{F}_{1}$ s were similar to the female parent in branching pattern and growth habit, were tall having thick and waxy stem. Siliqua set was more than parents whereas seed set was lower (Fig. $5 b$ ). The cross of $B$. carinata $x B$. napus was like female parent in terms of growth habbit. Waxy stem and tall height was observed (Fig. 5c). Growth habit was like that of male parent, profused and appressed siliqua were observed and reduced siliqua size as compared to the female parent in B.carinata $x$ B.nigra cross (Fig. $5 \mathrm{~d}$ ). B.napus $x$ B.carinata $\mathrm{F}_{1} \mathrm{~s}$ were closely resembled the female parent in plant type and growth habit. Pod and pod bearing habit was also like that of female parent (Fig. 5e). In B. napus $\times$ B. juncea cross the hybrids resembled the female parent in plant type and growth habit. The pods of the hybrids and its pod bearing habit also resembled the female parent B. napus (Fig. $5 f$ ). The $F_{1} s$ of $B$. junceax B. napus were quite vigorous, bushy and intermediate to their parents for inflorescence and other morphological attributes. The flowers with deep yellow corolla were intermediate in size. The anthers were rudimentary and were sterile. The traits viz. plant height, primary and secondary branches were more pronounced in the hybrids as compared to the parents. The siliquae resembled the female parent $B$. juncea. No seed set occurred on self-pollination.

(e) Meiotic irregularities in the interspecific hybrids: Meiotic analysis of the hybrids was characterized by irregularities at different phases of the cell cycle. Different irregularities viz., Improper alignment of chromosomes at Metaphase I, Improper alignment of chromosomes at Metaphase II, Laggards at Anaphase I, Scattering of chromosomes at Anaphase II, Bridge fragment configuration at Anaphase and Micronuclei at Telophase were recorded.(Fig. 6)

\section{Discussion}

The percent pollen germination in self was early as compared to the interspecific hybrids (Sridevi and Sarla, 1996). Pollen tube growth was fast in crosses where there was genome sharing between crosses. Rate of pollen tube growth was almost constant after $2 \mathrm{hrs}$ and $8 \mathrm{hrs}$ of pollination. An increase in the growth of pollen tube was observed from $24 \mathrm{hrs}$ to $48 \mathrm{hrs}$ of pollination. Pollen tube abnormality percent was high in distant crosses. The type of abnormality was not specific to a particular cross but all of the above mentioned abnormalities were recorded in each of the interspecific crosses made.No significant trend was observed in pollen tube abnormality with respect to time interval after pollination. Seed set percent was high in cases where the amphidiploid was crossed with another amphidiploid followed by amphidiploid $\times$ diploid and diploid $\times$ diploid crosses. Interspecific hybrids mostly showed an intermediate condition, but in some cases the predominance of maternal effect was observed (Song et al., 1993; Hu et al., 1992; Malek, 2007; Fu et al., 2018). High pollen germination percent was associated with better siliqua set percent. Likewise higher the mean pollen tube growth was associated with higher siliqua set percent. Higher pollen abnormality percent resulted in low siliqua set percent which indicated the failure of interspecific cross (Saba, 2012). Observationsof callose response, pollen germination, pollen tube growth, embryo development, siliqua set were the determinant of the crossability of any two parents (Bhat and Sarla, 2004; Rai et al 2012). The success rate was better when the amphidiploid species were used as a female parent (Kerlan et al., 1992; Choudhary and Joshi, 2012). Interspecific hybrids mostly showed an intermediate condition between the two parental species but in some of the hybrids the predominant effect of maternal parent was also visible (Song et al., 1993; Hu et al., 1992; Malek, 2007; Fu et al., 2018). The meiotic analysis of the F1's was characterized by irregularities in metaphase I, metaphase II, anaphase I, anaphase II and telophase. Orientation of the chromosomes at metaphase I and disjunction of chromosome at anaphase I and II was found abnormal (Meng et al., 1998; Souza, 1999; Gupta et al., 2014). In addition to these bridge-fragment configuration was also observed at anaphase I and anaphase II (Choudhary et al., 2012). As per the observations $B$. carinata performed better as a seed parent with $B$. nigra, $B$. juncea and $B$. rapa whereas, with $B$. napus it performed good as a pollen parent.

\section{Conclusion}


The crosses having the highest siliqua set percent had highest seed set percent. Overall the hybrids showed intermediate character, but predominance of maternal character was also found.Meiotic abnormalities in the form of laggards, micronuclei formation and misalignment of chromosomes were observed.Pre-fertilization abnormalities were found but still possibility of post fertilization barrier can also be explored. The study performed will facilitate breeders to select parents and direction of crosses for efficient gene transfer. Mostly the species with high chromosome number served well as female parent. The crosses where species with genome sharing were involved gave more successful results. Such studies will help to create novel genetic variations for the crop improvement programme and the evolution and speciation of the species can be better understood.

\section{Declarations}

None of the authors has any conflict issue.

\section{References}

1. Bhat, S. and Sarla, N. 2004. Identificationand overcoming barriers between Brassica rapaL. em. Metzg. and Brassica nigra (L.) Koch, crosses for the resynthesis of B. juncea(L.) Czern. Genet. Resour. Crop Evol.,51:455-469.

2. Choudhary, B. R. and Joshi, P. 2012. Cytomorphology of Brassica napus $\times$ B. rapahybrids and patterns of variation in the $F_{2}$ derivatives. Caryologia, 65:4, 316-321.

3. Fu, S., Yin, L., Xu, M., Li, Y., Wang, M., Yang, J., Fu, T., Shen, J., Ali, A., Zou, Q., Yi, B., Wen, J., Tao, L., Kang, Z. and Tang, R. 2018. Maternal double haploid production in interploidy hybridization between Brassica napus and Brassica allooctaploids. Planta, Volume 247, pp 113-125.

4. Gupta, M., Atri, C. and Banga, S.S. 2014.Cytogenetic stability and genome size variations in newly developed derived Brassica juncea allopolyploid lines. Journal of Oilseed Brassica, 5(2): 118-127

5. Hu, W.F.; Meng, J.L. and Liu, X.L. 1992. Effects of the genotype of Brassica napus on its crossability with B. carinata. Oil Crops of China, 2:18-22

6. Kerlan,M.C., Chevre, A.M., Eber, F., Baranger, A. and Renard, M. 1992. Risk assessment of outcrossing of transgenic rapeseed to related species: I. Interspecific hybrid production under optimal conditions with emphasis on pollination and fertilization. Euphytica, 62:145-153.

7. Malek, M.A. 2007. Study of crossability and $\mathrm{F}_{1}$ of interspecific hybridization between Brassica rapa (B. campestris) and B. nigra. Bangladesh J. Agric. Res., 32(3): 445-449.

8. Meng, J., S. Shi, L. Gan, Z. Li \& X. Qu. 1998. The production of yellow seeded Brassica napus (AACC) through crossing inter-specific hybrids of $B$. campestris (AA) and B. carinata(BBCC), with B. napus. Euphytica,103: 329-333.

9. Rai, P.K., Siddiqui, S.A., Singh, B.K. and Singh, V.V. 2012. Studies on pollen-pistil interaction in intergeneric and interspecific crosses of oilseed Brassicas and related genera. Vegetos, 25(2): 276-281.

10. Saba, I.2012 Studies on crossability among seven species of genus Brassica. Thesis submitted to GBPUA\&T, pp1-48.

11. Song, K., Tang. K. and Osborn, T.C. 1993. Development of synthetic Brassica amphidiploids by reciprocal hybridization and comparison to natural amphidiploids. Theor. Appl. Genet. 86:811-821.

12. Souza, A.M., Pagliarini, M.S. and Carraro, I.M. (1999). Abnormal spindles in second meiosis and its consequences in canola (Brassica napus and Brassica campestris). Braz. Arch. Biol. Technol.42: 47-52.

13. Sridevi.O. and Sarla, N. 1996. Reciprocal hybridization between Sinapis alba and Brassica species. Cruciferae Newsl. Eucarpia.,18(3):116-121.

14. Piotr Kaminski, Agnieszka Marasek-Ciolakowska, Małgorzata Podwyszynska, Michał Starzycki, El 'zbieta Starzycka-Korbasand Katarzyna Nowak (2020). Development and Characteristics of Interspecific Hybrids between Brassica oleracea L. and B. napus L. Agronomy 2020, 10, 1339

15. Katche, E.; Quezada-Martinez, D.; Ihien Katche, E.; Vasquez-Teuber, P.; Mason, A.S. (2019) Interspecific hybridization for Brassica crop improvement. Crop. Breed. Genet. Genom. 1, e 190007.

\section{Figure}

Figure 4 not available with this version.

\section{Figures}



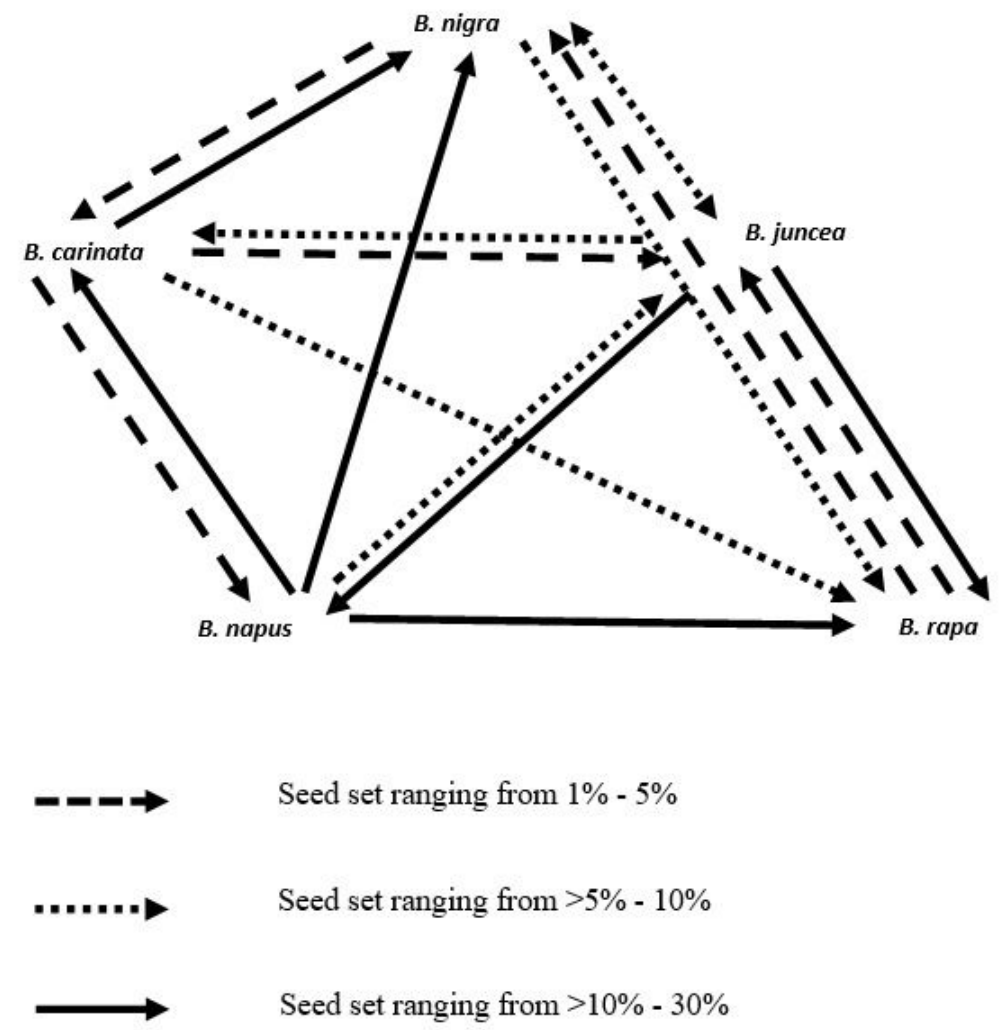

Figure 1

Diagram representing crossability relationship among Brassica species (based on percent seed set).

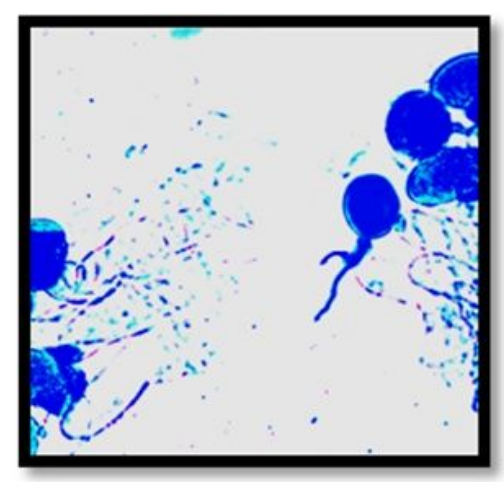

$\mathbf{a}$

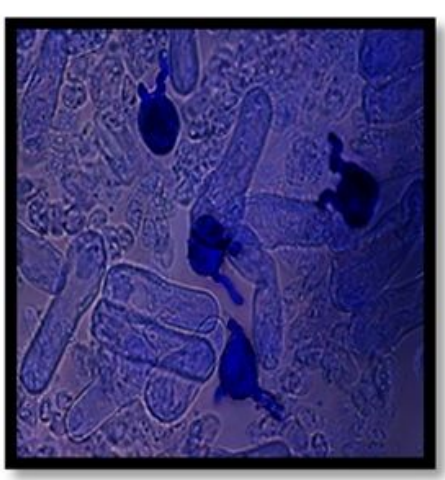

b

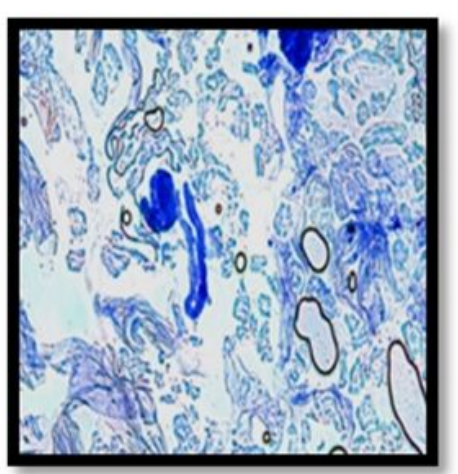

c

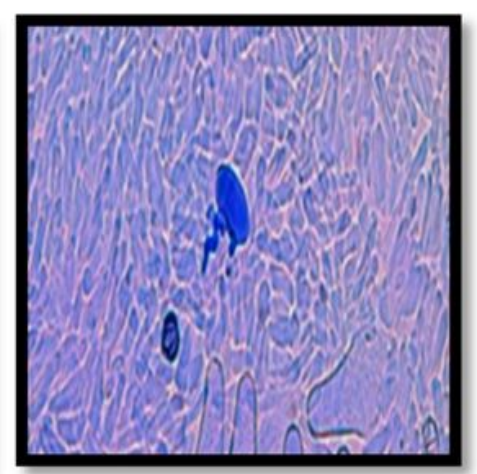

d

Figure 2

Bright Field Microscopy (a) branching of pollen tube (b) more than one pollen tube from a pollen (c) hairpin bend of pollen tube and (d) zig zag pollen tube 


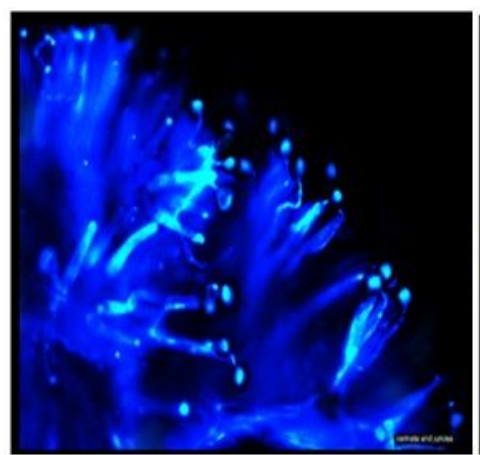

a

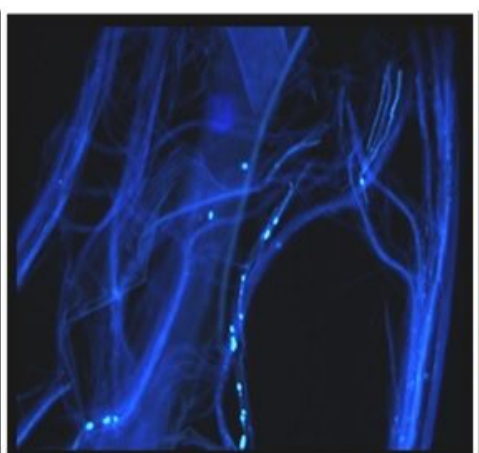

b

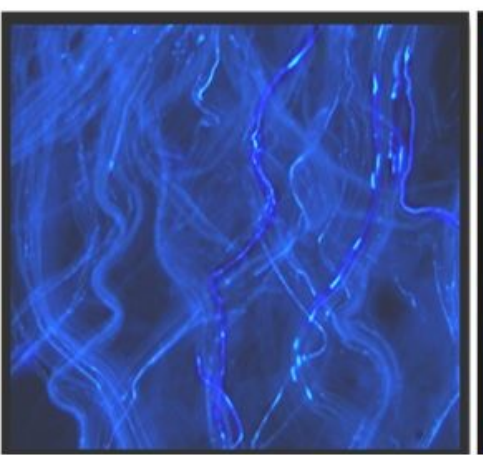

c

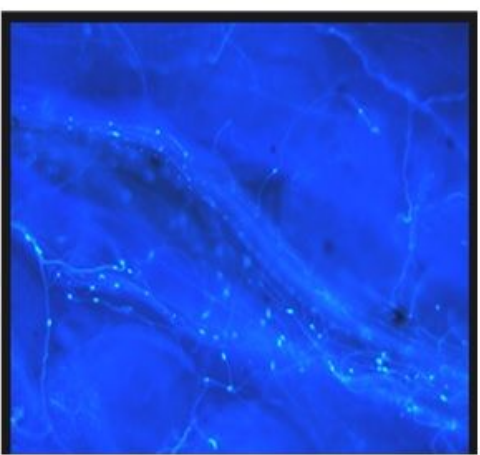

d

Figure 3

Fluroscent Microscopy (a) pollen tube on stigmatic surface (b) broken pollen tube (c) tangled pollen tube in mid styler region (d) pollen tube bypassing ovule

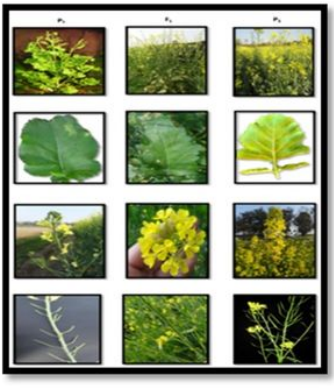

a

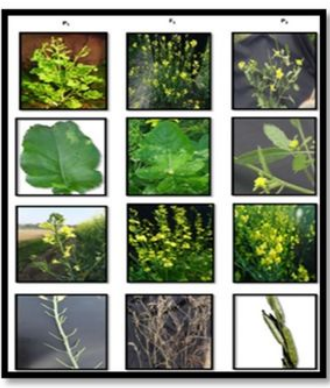

d

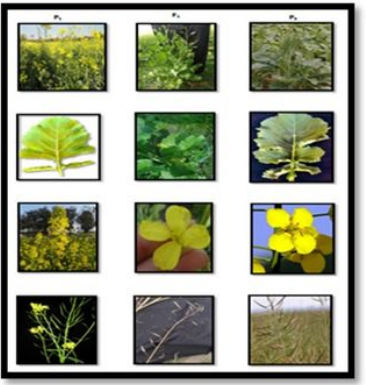

(g)

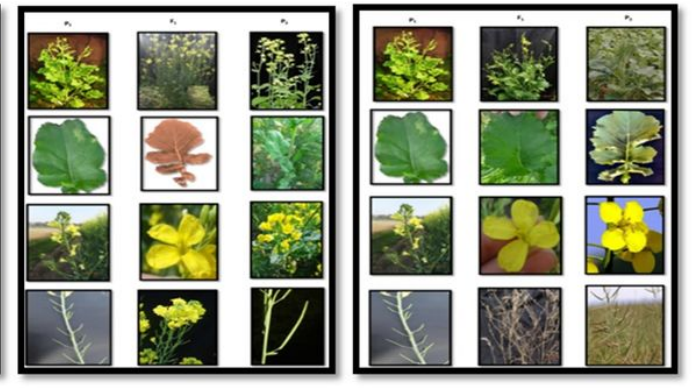

b

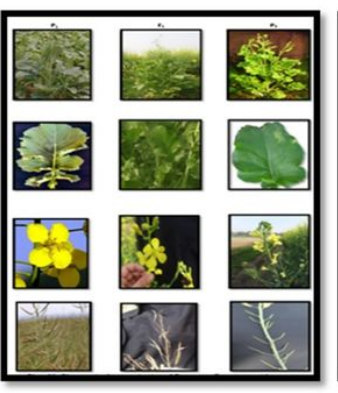

e

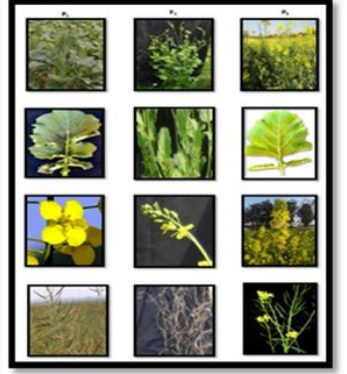

f

\section{Figure 4}

morphological Characterization of F1s (a)B.carinata X B.juncea (b)B.carinataX B. rapa (c)B. carinataX B. napus (d)B.carinata X B. nigra (e)B. napus X B. carinata (f) B.napus X B.juncea(g) B.juncea X B.napus 


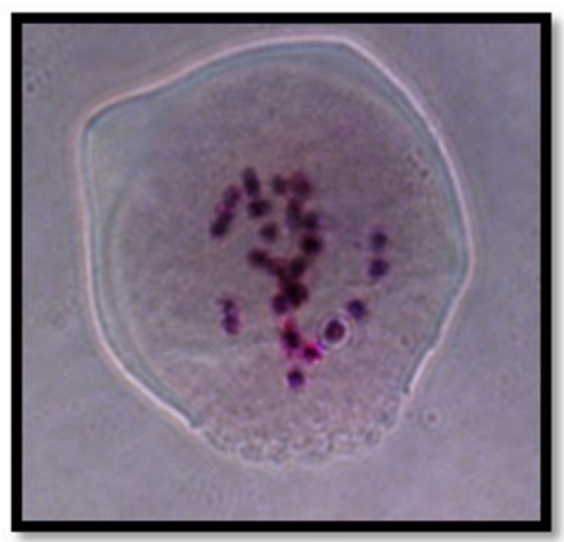

a

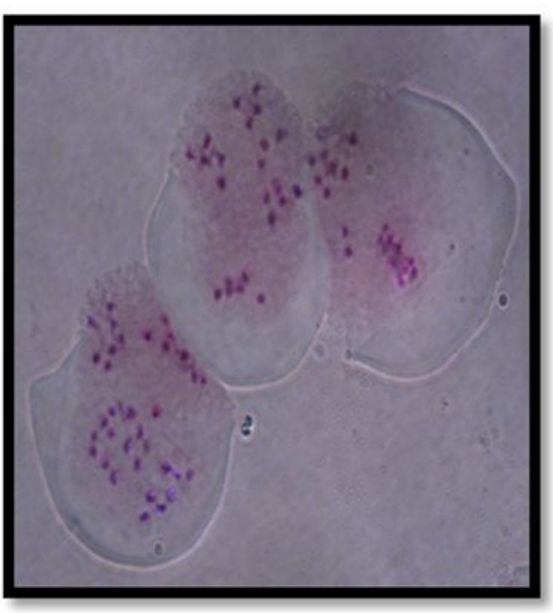

d

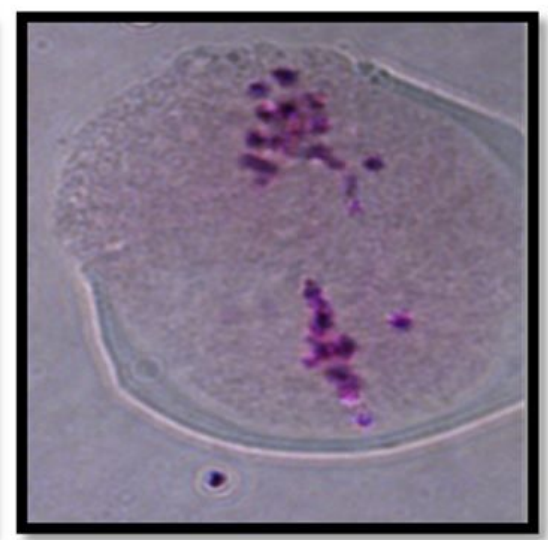

b

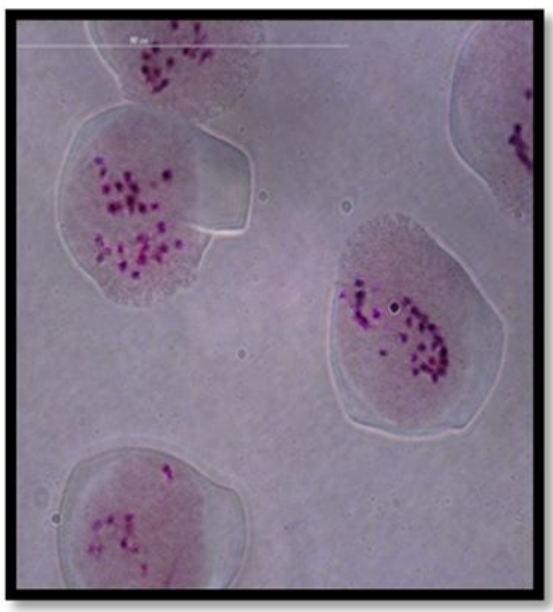

$\mathbf{e}$

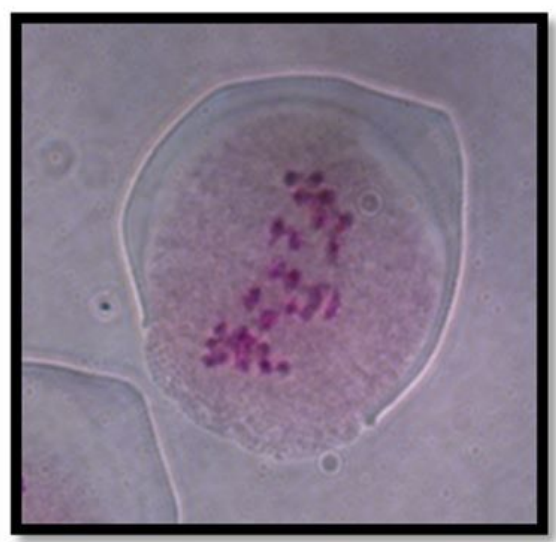

c

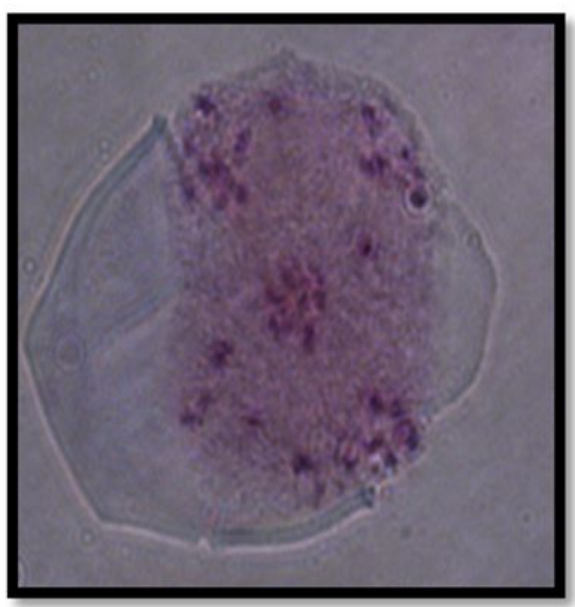

f

Figure 5

Meiosis studies (a)Improper alignment of chromosomes at Metaphase I (b)Improper alignment of chromosomes at Metaphase II (c)Laggards at Anaphase I (d)Scattering of chromosomes at Anaphase II (e)Bridge fragment configuration at Anaphase (f)Micronuclei at Telophase 